\title{
Underground Sagnac gyroscope with sub-prad/s rotation rate sensitivity: Toward general relativity tests on Earth
}

\author{
Angela D. V. Di Virgilio $\odot,{ }^{1}$ Andrea Basti $\odot,{ }^{2}$ Nicolò Beverini $\odot,{ }^{2}$ Filippo Bosi,,${ }^{1}$ Giorgio Carelli $\odot,{ }^{2}$ Donatella Ciampini, ${ }^{2}$ \\ Francesco Fuso $\odot,{ }^{2}$ Umberto Giacomelli $\odot,{ }^{1}$ Enrico Maccioni, ${ }^{1,2}$ Paolo Marsili, ${ }^{2}$ Antonello Ortolan $\odot,{ }^{3}$ Alberto Porzio ${ }^{\circ},{ }^{4}$ \\ Andrea Simonelli, ${ }^{1}$ and Giuseppe Terreni ${ }^{1}$ \\ ${ }^{1}$ INFN Sezione di Pisa, Largo Bruno Pontecorvo 3, I-56127 Pisa, Italy \\ ${ }^{2}$ Dipartimento di Fisica Enrico Fermi, Università di Pisa, Largo Bruno Pontecorvo 3, I-56127 Pisa, Italy \\ ${ }^{3}$ INFN-National Laboratories of Legnaro, viale dell'Università 2, I-35020 Legnaro (PD), Italy \\ ${ }^{4}$ CNR-SPIN and INFN, Napoli, Complesso Universitario di Monte Sant'Angelo, via Cintia, I-80126 Napoli, Italy
}

(Received 26 March 2020; accepted 4 August 2020; published 17 September 2020)

\begin{abstract}
Measuring in a single location on Earth its angular rotation rate with respect to the celestial frame, with a sensitivity enabling access to the tiny Lense-Thirring effect, is an extremely challenging task. GINGERINO is a large frame ring laser gyroscope, operating as free running and unattended inside the underground laboratory of the Gran Sasso, Italy. The main geodetic signals, i.e., annual and Chandler wobbles, daily polar motion, and length of the day, are recovered from GINGERINO data using standard linear regression methods, demonstrating a sensitivity approaching tens of frad/s, therefore close to the requirements for Earth-based Lense-Thirring and Lorentz violation tests.
\end{abstract}

DOI: 10.1103/PhysRevResearch.2.032069

Sensing the rotation rate is essential for both applications and fundamental science. Ring laser gyroscopes (RLGs), based on the Sagnac effect, have been established as the top sensitivity instruments for measuring rotation rates relative to an inertial frame with excellent accuracy [1]. Further to the geodetic information relating to its instantaneous rotation, knowing the absolute value of the Earth's rotation rate and investigating its variations with an Earth-based instrument are of paramount interest to detect relativistic effects, e.g., the Lense-Thirring one [2-8]. Improving the accuracy and reliability in data analysis is a crucial point for enabling the use of RLGs in general relativity (GR) [9] Earth-based measurements and in the investigation of new physics theories [10-12]. Recently, it has been pointed out that RLG can effectively contribute to the Lorentz violation quest $[13,14]$. Among geodetic effects, the polar motion, mainly composed of daily variations, and the annual and Chandler wobbles have been already observed by monolithic RLG [15]. Polar motion has been analytically modeled in terms of its local effects as a function of time, latitude, and longitude on a RLG [16]. The wobbles are routinely and constantly measured by IERS (the International Earth Rotation and Reference Systems Service) on a daily basis [17]. Moreover, variations of the length of the day (LOD) affect the Earth's angular rotation rate $\Omega_{\oplus}$ via a term defined as $\Delta \omega_{3}$. Therefore, the Sagnac frequency $f_{s}$ measured by an RLG is affected by both variations of the

Published by the American Physical Society under the terms of the Creative Commons Attribution 4.0 International license. Further distribution of this work must maintain attribution to the author(s) and the published article's title, journal citation, and DOI. absolute value of the angular rotation rate and of its projection, which cannot be disentangled from each other by a single RLG [6]. RLGs are, however, considered the only instruments able to provide almost real-time subdaily measurements of the relevant quantities $[1,18]$.

In this Rapid Communication we demonstrate that the sensitivity of a heterolithic RLG, GINGERINO, can be pushed to the envelope of the GR sensitivity region by applying statistical methods to the analysis of its data. GINGERINO $[19,20]$ is a 3.6-m-side RLG in continuous, unattended operation inside the underground Gran Sasso laboratory (LNGS, Italy) and is able to provide data with a duty cycle $\gtrsim 80 \%$ [21]. The purpose of the statistical methods is to look for evidence of geodetic signals, $F_{\text {geo }}$, in the acquired data. A linear regression procedure evaluates the parameters' weighting contribution of different signals accounting for laser dynamics, information from a colocated tilt meter, and environmental probes (local temperature and tides), in order to find the best estimate of the expected geodetic signals $F_{\text {IERS }}$.

The signal of interest in the analysis is the Sagnac frequency $f_{s}$, which is proportional to the total angular velocity $\vec{\Omega}_{T}$ according to $[5,6]$

$$
\begin{aligned}
f_{s} & =S \Omega_{T} \cos \beta, \\
S & =4 \frac{A}{L \lambda},
\end{aligned}
$$

where $S$ is the scale factor depending on the area $A$ enclosed in the cavity, its perimeter $L$, and the laser wavelength $\lambda$, while $\beta$ is the angle between the area vector and $\vec{\Omega}_{T} . \vec{\Omega}_{T}$ is the total angular velocity with respect to an inertial frame experienced by the gyroscope optical cavity, resulting from the sum of several terms: the dominant one, the Earth's angular 
rotation rate $\vec{\Omega}_{\oplus}$, and the local and instrumental rotations, in principle unknown, defined as $\vec{\omega}_{L}$. The Sagnac frequency $f_{s}$ is also sensitive to fluctuations of the angle $\beta$ due to polar motion and to local and instrumental tilting. Accordingly, we can consider the Earth's angular rotation $\Omega_{\oplus}=\bar{\Omega}_{\oplus}+\Delta \omega_{3}+$ $\Omega_{\mathrm{GR}}$, where $\bar{\Omega}_{\oplus}$ indicates the nominal value [17], $\Delta \omega_{3}$ accounts for low-frequency variations of the Earth's rotation rate connected to the changes of LOD and to zonal tides, and $\Omega_{\mathrm{GR}}$ accounts for the GR effects, due to the fact that GINGERINO is connected to the Earth's crust and operates in a rotating noninertial frame. At the latitude of the Gran Sasso underground laboratory, for a RLG lying in a horizontal plane the effect of $\Omega_{\mathrm{GR}}$ is expected to be $2.29 \times 10^{-14} \mathrm{rad} / \mathrm{s}$, corresponding, for the case of GINGERINO, to a shift in $f_{s}$ of $0.130 \mu \mathrm{Hz}$ [5]. Instrumental and local effects have to be integrated in the analysis. Assuming the scale factor $S$ to be constant, which is reasonable in a first approximation owing to the large temperature stability of the underground laboratory (typical amplitude fluctuations are of the order of $0.1{ }^{\circ} \mathrm{C}$ in 1 month) and the small thermal expansion of the granite-made gyroscope frame $\left(6.5 \times 10^{-6}{ }^{\circ} \mathrm{C}^{-1}\right)$, the instrumental and local changes in the rotation velocity $\omega_{L}$ and variations of the absolute orientation $\delta \theta_{L}$ can be included in Eq. (1). First-order expansion leads to an effective Sagnac frequency $F_{\text {eff }}$,

$$
\begin{aligned}
F_{\text {eff }}= & S\left[\sin \theta\left(\Delta \omega_{3}+\omega_{L}+\bar{\Omega}_{\oplus}+\Omega_{\mathrm{GR}}\right)\right. \\
& \left.+\cos \theta\left(\delta \theta_{L}+P M\right)\left(\Delta \omega_{3}+\omega_{L}+\bar{\Omega}_{\oplus}+\Omega_{\mathrm{GR}}\right)\right],
\end{aligned}
$$

where $P M$ represents the combined effects of polar motion and of the annual and Chandler wobbles. The first term in Eq. (2) depends on the actual changes of the angular velocity, while the second one is due to changes in the projection. $F_{\text {eff }}$ can be decomposed as a sum of $F_{\text {IERS }}$ and of the local $F_{L}$,

$$
\begin{gathered}
F_{\mathrm{IERS}}=S\left[\left(\Delta \omega_{3}+\bar{\Omega}_{\oplus}+\Omega_{\mathrm{GR}}\right) \sin \theta\right. \\
\left.+P M\left(\Delta \omega_{3}+\bar{\Omega}_{\oplus}+\Omega_{\mathrm{GR}}\right) \cos \theta\right], \\
F_{L}=S\left[\omega_{L} \sin \theta+P M \omega_{L} \cos \theta\right. \\
\left.+\delta \theta_{L}\left(\Delta \omega_{3}+\omega_{L}+\bar{\Omega}_{\oplus}+\Omega_{\mathrm{GR}}\right) \cos \theta\right] .
\end{gathered}
$$

Using the available data, $F_{\text {IERS }}$ has been evaluated for the gyroscope location [22]. The main purpose of the linear regression is to compare the Sagnac frequency of the gyroscope with the expected $F_{\text {IERS }}$, identifying the local signals $F_{L}$ by means of the available environmental probes. The evaluation of $\delta \theta_{L}$ is based on the two-channel tilt meter located on top of the RLG monument. Using both channels $\left(\delta \theta_{L 1}\right.$ and $\left.\delta \theta_{L 2}\right)$ enables accounting for the motion of the RLG cavity due to local geophysical and instrumental effects, where rotations and tilts are mixed together, thus making it possible to reconstruct $\omega_{L}$ in Eq. (3). Following Eq. (3), the effects of changes in the projection are evaluated by the product between $\delta \theta_{L}$ and the total rotation velocity $\left(\Delta \omega_{3}+\omega_{L}+\bar{\Omega}_{\oplus}+\Omega_{\mathrm{GR}}\right)$. The latter must be determined by iterating the linear regression procedure, starting with an average estimation of the angular velocities and leading to convergence typically in a couple of iterations. The projection through $\cos \theta$ of the term $P M$, accounting for the combined effects of polar motion and annual and Chandler wobbles, can also be determined following a similar iterative procedure. Data from a temperature sensor

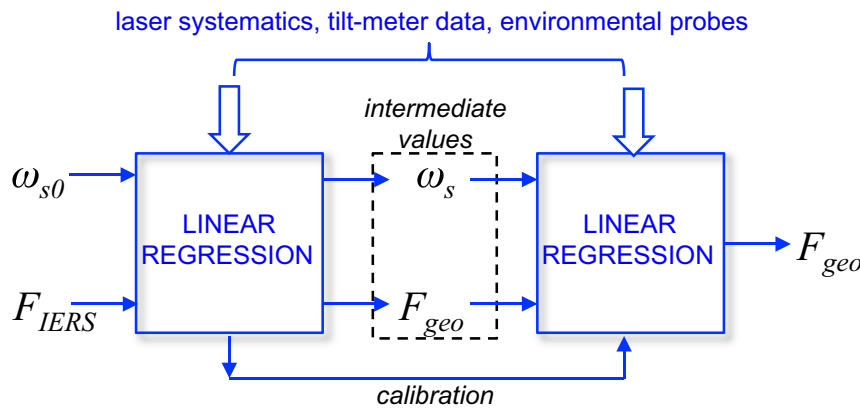

FIG. 1. Sketch of the linear regression procedure. In the first step the initial evaluation of the Sagnac angular frequency $\omega_{s 0}$ and the $F_{\text {IERS }}$ data represent the input of the linear regression, along with a vector containing all terms related to laser systematics, tilt-meter signals, and environmental probes. An intermediate value of $\omega_{s}$ and an intermediate estimate of $F_{\text {geo }}$ are obtained at the output, along with a factor accounting for the cross calibration of the Sagnac frequency with $F_{\text {IERS }}$. In the subsequent step the refined estimate of $F_{\text {geo }}$ is obtained, accounting for cross calibration. The procedure is iterated up to convergence.

are included in the linear regression, although their effect is rather small. It has been checked that pressure variations, measured by dedicated probes, do not play a significant role. The Sagnac angular frequency $\left(\omega_{s}=2 \pi f_{s}\right)$ can be determined from the measured beat note and the output power of the laser beams counterpropagating in the RLG cavity, according to the recently developed approach described in Refs. [23,24]: The first step of the analysis removes the backscatter noise providing an initial evaluation of the Sagnac angular frequency $\omega_{s 0}$, and the second step eliminates laser dynamics. Briefly, the approach accounts for the occurrence of technical issues such as those related to dark currents in the photodetectors, those associated with the laser operation in the two counterpropagating beams, and the related losses, via different correcting terms, denoted as $\omega_{\xi}, \omega_{n s 1}, \omega_{n s 2}$, and specifically defined form factors. Since the linear regression procedure leading to $F_{\mathrm{geo}}$ involves both ac and dc terms, the latter including the abovementioned $\omega_{\xi}, \omega_{n s 1}, \omega_{n s 2}$, and $\bar{\Omega}_{\oplus}+\Omega_{\mathrm{GR}}$, a cross calibration of $f_{s}$ with IERS data is needed, which is accomplished at a single, arbitrarily chosen data point. It has been checked that the results do not depend on the choice of the calibration point. In the calculation, a scale factor $S=5.6890 \times 10^{6} \mathrm{rad} / \mathrm{s} / \mathrm{Hz}$ is used, whose accuracy is dictated by the machining precision (tenths of a millimeter) of the monument supporting the RLG. $S$ and the projection angle are multiplicative factors, which cannot be distinguished from each other. It is assumed that the uncertainty in the proportionality constant is mainly due to the orientation of the cavity, in particular to the deviations of its plane with respect to the horizontal one. The latitude of the underground laboratory is $0.7409 \mathrm{rad}$, whereas the analysis indicates a value of $0.7420 \mathrm{rad}$, corresponding to an extra $\simeq 1$ mrad in the absolute orientation of the ring cavity.

The general scheme of the analysis is shown in Fig. 1, which follows the approach that we have recently developed $[23,24]$. Two distinct methods have been applied to the linear regression procedure. In the first one, all available data are used at once. In the second method, linear regression is carried 


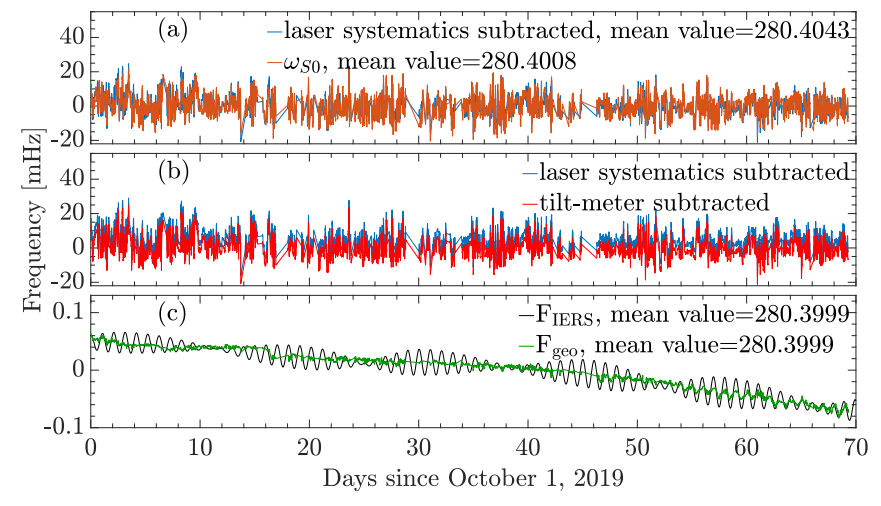

FIG. 2. Progress of the linear regression procedure (single-fit method): (a) $\omega_{s 0}$ and $F_{\text {eff }}$ obtained by subtracting effects related to laser systematics; (b) $F_{\text {eff }}$ after further subtraction of the effects related to tilt-meter signals; (c) the evaluated $F_{\text {geo }}$ compared with

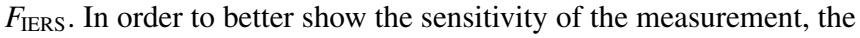
plotted data are subtracted for the mean values, as reported in the legends in Hz. Note that only data selected for the analysis are shown in the graph, where missing data are replaced by straight lines.

out on separated time windows consisting of three days each, but keeping only the central two days in the final result. Overlapping and smoothing were not applied. Parameters resulting from the linear regression of the whole data set are used as the initial guess in the above-described procedure, in particular the guess of $\omega_{s}$, accounting for the contribution of the terms describing the projection by the angle $\delta \theta_{L}$ in Eq. (3). In each window the cross-calibration procedure is repeated. Two independent and very different sets of data have been analyzed: 30 days from 16 June 2018, and 70 days from 1 October 2019. Data pertaining to 2018 showed a higher contrast in the Sagnac interferogram and a higher duty cycle, since the temperature was a factor of 10 more stable than for the 2019 data set. Approximately $85 \%$ and $79 \%$ of the data have been selected, respectively.

Figure 2 demonstrates the effect of correcting data of the 2019 set for laser systematics [Fig. 2(a)] and for $\delta \theta_{L}, \omega_{L}$ [Fig. 2(b)]; the effects of temperature variations are not shown since they are at the level of $10 \mu \mathrm{Hz}$, and not well visible in the graphs. Figure 2(c) shows the final result, $F_{\text {geo }}$, as obtained with the single-fit method, compared with $F_{\text {IERS. The annual }}$ and Chandler wobbles effect, reflected in the slow trend of data, is well reproduced in the single fit. On the contrary, the approach turns out not to be sufficient for retrieving the daily polar motion, which is barely visible in Fig. 2(c). The 2018 data set shows similar behavior: The laser systematics and the tilt-meter corrections $\delta \theta_{L}$ and $\omega_{L}$ take into account most of the disturbances. Remarkably, despite the qualitative difference between the two data sets, the evaluations exhibit very similar uncertainties: Root-mean-square (rms) errors of $10.8 \mu \mathrm{Hz}$ and $10.7 \mu \mathrm{Hz}$ are obtained. Typically, the covariance test ANOVA gives high $F$ statistics versus a constant model and a $p$ value close to zero. The inadequate reproduction of the daily polar motion achieved with the single-fit method is not surprising, since polar motion is a periodic signal with a null mean value for a long-time average. Moreover, the model adopted in the analysis can turn out to be oversimplified, since

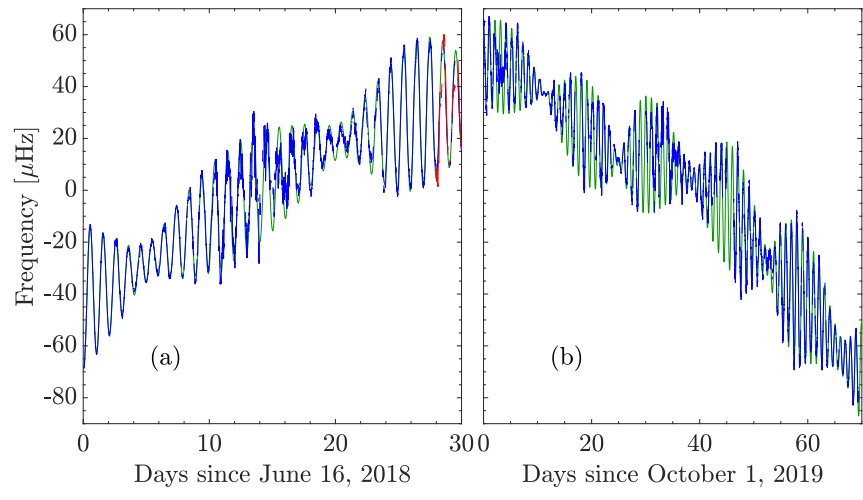

FIG. 3. The evaluated $F_{\text {geo }}$ compared with $F_{\text {IERS }}$ (blue and green lines, respectively) for (a) the 2018 and (b) the 2019 data sets. Uncertainties are below $1 \mu \mathrm{Hz}$ (equivalent to an error in the evaluated angular rotation rate of $1.7 \times 10^{-13} \mathrm{rad} / \mathrm{s}$ ) in a bandwidth corresponding to a $600 \mathrm{~s}$ measurement time. An evaluation is carried out with the separated time window method. Data marked in red in (a) demonstrate the predictive abilities of the approach, as discussed in the text. In both panels the mean values have been subtracted, and error bars not visible in this scale.

it accounts only for daily variations, while it is well known that other very similar signals are present in that frequency bandwidth [16]. The analysis is clearly improved when the fit is carried out over the separated time windows [25]. In particular, the daily polar motion is correctly recovered, as demonstrated in Fig. 3, referring to the 2019 [Fig. 3(a)] and 2018 [Fig. 3(b)] data sets. It must be noted that the observed daily variation has been carefully checked not to stem from disturbances, e.g., daily noise due to anthropic activity, which is strongly suppressed in the underground laboratory. Moreover, although the data statistics is not large enough to precisely discriminate the frequency of the daily polar motion from tides, it has been verified that the reconstructed signal is constantly in phase with the one expected for the polar motion. Remarkably, the approach shows also predictive capabilities. Indeed, by fitting on the separated time windows over a certain subset of available data, the behavior for a subsequent subset can be reliably predicted. The reconstructed signal in Fig. 3(a) for the last two days, marked in red, has been produced by using the linear regression in the previous period.

$\Delta \omega_{3}$, i.e., the low-frequency variations of the Earth's rotation rate connected to changes of LOD and to zonal tides, is provided by IERS with two options, with or without zonal tides. Its estimate based on GINGERINO data can be obtained by comparing the results obtained by including, or not, its contribution in the linear regression procedure. Figure 4 shows the evaluated $\Delta \omega_{3}$ for the 2019 data set using both the mentioned options; for the 2018 data, please see the Supplemental Material [25]. A comparison of the results obtained from GINGERINO and IERS data leads to a standard deviation of the residual $\simeq 0.2 \mathrm{prad} / \mathrm{s}$. The agreement is even better when the effect of zonal tides is evaluated by arranging the results of different analyses. We remark that, in the case where $F_{\text {geo }}$ is affected by unidentified disturbances not included in the model, their effect would be subtracted in the evaluation of $\Delta \omega_{3}$, with them being present in both evaluations. The found 


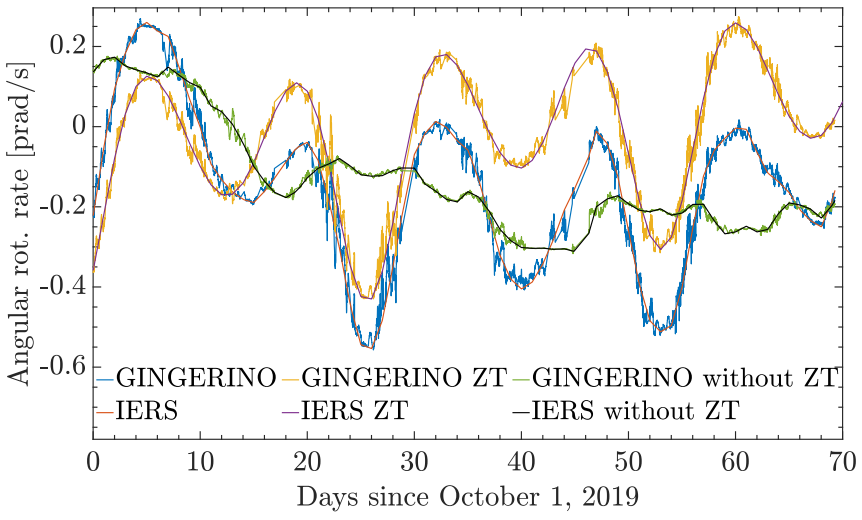

FIG. 4. Effects of the low-frequency variations $\Delta \omega_{3}$ on the Earth's angular rotation rate evaluated from the GINGERINO data and compared with IERS data. A comparison is carried out both including and neglecting contributions from the zonal tides, as discussed in the text. The 2019 data set is considered.

values of $\Delta \omega_{3}$ are close to the noise floor of the apparatus. In this case the method is not predictive and provides mainly compatibility between the model and available data. Moreover, to check the validity of the analysis, synthetic signals, either chirped or periodic at low frequency, of the same order of magnitude of $\Delta \omega_{3}$ have been used. When added to both the data and the model, such synthetic signals have been correctly identified. In general, the analysis leads to larger errors when signals are inserted in the model only, but not always a signal present in the model and not added to the data is recognized as a fake. To improve the validity of the analysis for such small signals it will be necessary to improve both the calibration of the RLG and the model, in order to achieve a more effective rejection of local disturbances.

The feasibility of the GR test has been checked comparing to the results obtained with different models: The difference of the average values of $F_{\mathrm{IERS}}$ including and neglecting $\Omega_{\mathrm{GR}}$ is consistent with the expected GR shift. Since $\Omega_{\oplus}$ is rather stable in time, by analogy with the clock frequency analysis the quantity relevant for assessing the quality of the approach and of the produced results is the Allan variance, in particular the modified Allan deviation (MAD) [26], which provides also indications on the nature of the noise limiting the measurement accuracy. Figure 5 shows the MAD calculated for $\omega_{s 0}$, for $F_{\text {geo }}$ and for the residuals, defined as $\left(F_{\text {geo }}-F_{\text {IERS }}\right)$. The different MAD found for $\omega_{s 0}$ in the two data sets confirms the already-mentioned differences between them. The longtime behavior very well follows the IERS data for both data sets, as highlighted by the consistent decrease of the residual MAD with time. The peak around 3-4 $\times 10^{4} \mathrm{~s}$ indicates the occurrence of other signals at that frequency that have not been included in the model or cannot be retrieved by using only tilt-meter data. The behavior shown in Fig. 5 suggests that shot noise plays a dominant role in the measurement, while being larger than the theoretical shot noise, evaluated for GINGERINO below $1 \mathrm{prad} / \mathrm{s}$ in $1 \mathrm{~s}$ measurement time. The tilt meter is not the best suited instrument to monitor $\delta \theta_{L}$ since it provides it with respect to the local vertical, while the analysis would need changes with respect to the rotation

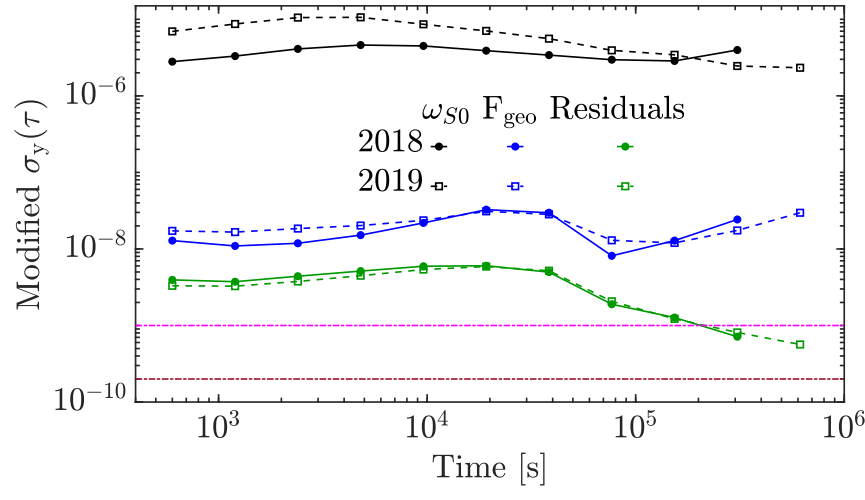

FIG. 5. Modified Allan deviation $\sigma_{y}(\tau)$ calculated for $\omega_{s 0}$, for $F_{\text {geo }}$, and for the residuals defined as in the text. Both 2019 (dotted lines) and 2018 (solid lines) data sets are shown. Error bars are not visible in this scale. The two horizontal dotted-dashed lines indicate the two levels that are meaningful for Lorentz violation and LenseThirring tests, the top one being the first one.

axis of $\vec{\Omega}_{T}$. We remark that the availability of an array of RLGs, as foreseen in the GINGER project, would not make it necessary to use the tilt-meter data. In particular, the problem of evaluating $\delta \theta_{L}$ would be fully solved by using a pair of RLGs, with one oriented at the maximum Sagnac signal, or independently measuring the relative angle between the two RLGs [6].

It is important to remark that the level of sensitivity of 1 part in $10^{9}$ of the Earth's rotation rate would provide interesting measurements of two Lorentz-violating terms in the framework of the standard-model extension [13]. In one case, sensitivities that are competitive with recent laboratory [27,28] and perhaps solar system [29] tests would result. For the other term, measurements competitive with the best existing limits, which currently come from radio pulsar studies [30], would result.

It is therefore demonstrated that with a minimum square linear regression procedure, taking into account the expected $F_{\text {IERS }}$, the laser dynamics, and the environmental monitors, it is possible to recover geodetic effects from GINGERINO data. In particular, $F_{\text {geo }}$ reproduces all its main features, such as annual and Chandler wobbles, daily polar motion, and the very low-frequency contribution $\left(\Delta \omega_{3}\right)$ due to LOD and zonal tides. The residuals show the occurrence of other daily and subdaily signals, which are absent in the used model containing only main contributions to the observed quantities. Remarkably, their MAD exhibits a decrease with the measurement time similar to the one shown by shot noise, and eventually drops down to $18 \mathrm{prad} / \mathrm{s} \mathrm{Hz}^{-1 / 2}$, approximately ten times above the theoretical shot noise of GINGERINO, which could be probably further reduced by getting rid of the tilt-meter signals in the linear regression, as enabled by an array of RLGs.

The analysis demonstrates the very high sensitivity which can be obtained by using RLGs-remarkably, Fig. 5 indicates a sensitivity of $40 \mathrm{frad} / \mathrm{s}$ with $3 \times 10^{5} \mathrm{~s}$ integration timeand points to the necessity of identifying and subtracting systematics associated with nonlinear laser dynamics. The 
underground location provides several advantages and sensitivity is shown to be adequate for the purpose of the GINGER project [6], where an array of RLGs, with an improved heterolithic structure and independent calibration strategies, is foreseen.

The cross calibration of the presently available GINGERINO data with IERS is effectively accomplished for $\Omega_{\oplus}$, paving the way for a reliable use of RLG data in the investigation of GR effects with Earth-based measurements.

We thank the Gran Sasso staff in support of the experiments, particularly Stefano Gazzana. We thank Ulli Schreiber for his continuous support in discussions and in favor to RLG. We thank Giancarlo Cella, Jay Tasson, and Roberto Devoti for useful discussions.
[1] K. U. Schreiber and J. P. R. Wells, Rev. Sci. Instrum. 84, 041101 (2013).

[2] I. Ciufolini and E. C. Pavlis, Nature (London) 431, 958 (2004).

[3] C. W. F. Everitt et al., Phys. Rev. Lett. 106, 221101 (2011).

[4] F. Bosi, G. Cella, A. Di Virgilio et al., Phys. Rev. D 84, 122002 (2011).

[5] A. Tartaglia, A. Di Virgilio, J. Belfi, N. Beverini, and M. L. Ruggiero, Eur. Phys. J. Plus 132, 73 (2017).

[6] A. D. V. Di Virgilio, J. Belfi, W.-T. Ni, N. Beverini, G. Carelli, E. Maccioni, and A. Porzio, Eur. Phys. J. Plus 132, 157 (2017).

[7] D. M. Lucchesi, L. Anselmo, M. Bassan, C. Pardini, R. Peron, G. Pucacco, and M. Visco, Classical Quantum Gravity 32, 155012 (2015).

[8] I. Ciufolini, A. Paolozzi, E. C. Pavlis et al., Eur. Phys. J. Plus 132, 336 (2017)

[9] M. O. Scully, M. S. Zubairy, and M. P. Haugan, Phys. Rev. A 24, 2009 (1981)

[10] S. Capozziello and M. D. De Laurentis, Phys. Rep. 509, 167 (2011).

[11] R. Aldrovandi and J. G. Pereira, Teleparallel Gravity: An Introduction (Springer, Berlin, 2013).

[12] T. Clifton, P. G. Ferreira, A. Padilla, and C. Skordis, Phys. Rep. 513, 1 (2012).

[13] S. Moseley, N. Scaramuzza, J. D. Tasson, and M. L. Trostel, Phys. Rev. D 100, 064031 (2019).

[14] V. A. Kostelecký and N. Russell, Rev. Mod. Phys. 83, 11 (2011).

[15] K. U. Schreiber, T. Klügel, J. P. R. Wells, R. B. Hurst, and A. Gebauer, Phys. Rev. Lett. 107, 173904 (2011).

[16] M. Tercjak and A. Brzeziński, Pure Appl. Geophys. 174, 2719 (2017).

[17] Series ECC Paris Observatory: IERS Orientation Center, https: //hpiers.obspm.fr/eop-pc/index.php.

[18] S. Böhm, M. Schartner, A. Gebauer, T Klügel, U. Schreiber, and T. Schüler, Adv. Geosci. 50, 9 (2019).
[19] J. Belfi, N. Beverini, F. Bosi et al., Rev. Sci. Instrum. 88, 034502 (2017).

[20] J. Belfi, N. Beverini, G. Carelli, A. Di Virgilio, U. Giacomelli, E. Maccioni, A. Simonelli, F. Stefani, and G. Terreni, Appl. Opt. 57, 5844 (2018).

[21] GINGERINO is in free-running mode, without geometry control; as a consequence, it exhibits sparse mode jumps and split mode operation leading to data unsuitable for the analysis, which are therefore discarded.

[22] M. Tercjak (private communication).

[23] A. D. V. Di Virgilio, N. Beverini, G. Carelli, D. Ciampini, F. Fuso, and E. Maccioni, Eur. Phys. J. C 79, 573 (2019)

[24] A. D. V. Di Virgilio, N. Beverini, G. Carelli, D. Ciampini, F. Fuso, U. Giacomelli, E. Maccioni, and A. Ortolan, Eur. Phys. J. C 80, 163 (2020).

[25] See Supplemental Material at http://link.aps.org/supplemental/ 10.1103/PhysRevResearch.2.032069 for details on the results with linear regression on shorted time windows, and for the evaluation of $\Delta \omega_{3}$ for the 2018 data set.

[26] W. Riley and D. A. Howe, Handbook of Frequency Stability Analysis, NIST Special Publication 1065 (NIST, Gaithersburg, MD, 2008), https://www.nist.gov/publications/handbookfrequency-stability-analysis.

[27] N. A. Flowers, C. Goodge, and J. D. Tasson, Phys. Rev. Lett. 119, 201101 (2017).

[28] K.-Y. Chung, S.-W. Chiow, S. Herrmann, S. Chu, and H. Muller, Phys. Rev. D 80, 016002 (2009); H. Müller, S.-w. Chiow, Q. Long, S. Herrmann, and S. Chu, Phys. Rev. Lett. 100, 180405 (2008).

[29] A. Bourgoin, C. Le Poncin-Lafitte, A. Hees, S. Bouquillon, G. Francou, and M.-C. Angonin, Phys. Rev. Lett. 119, 201102 (2017).

[30] L. Shao and Q. G. Bailey, Phys. Rev. D 98, 084049 (2018). 